\title{
CONSUMO DE AGUA DE OVINOS ALIMENTADOS CON DIFEREN- TES NIVELES DE NOPAL (OPUNTIA FICUS INDICA) EN BRASIL
}

\author{
WATER INTAKE IN SHEEPS FED DIFFERENT LEVELS OF PRICKLY PEAR \\ (OPUNTIA FICUS INDICA) IN BRAZIL \\ Costa, R.G. ${ }^{1 *}$, Hernandez, T.I. ${ }^{1 A}$, Medeiros, G.R. ${ }^{2}$, Medeiros, A.N. ${ }^{1}$, Azevedo, P.S. ${ }^{1}$, \\ Pinto, T.F. ${ }^{1}$ y Delgado, J.V. ${ }^{3}$
}

1Programa de Pós-Graduación en Zootecnia. UFPB. *betogermano@hotmail.com; Aisra_ht@hotmail.com 2Instituto nacional de Semiárido (INSA).Insa@insa.gov.br

32Departamento de Genética. Universidad de Córdoba. España. juanviagr218@gmail.com

\author{
PALABRAS CLAVE ADICIONALES \\ Cactácea. Corderos. Déficit hídrico. Producción.
}

\section{RESUMEN}

En 45 ovinos machos Santa Inés (peso vivo medio de $27,50 \pm 0,48 \mathrm{~kg}$ ) se evaluó el consumo de agua, al consumir raciones con niveles crecientes $(0,25,50,75,100 \%)$ de nopal (Opuntia fícus indica Mill) en sustitución al maíz molido. El diseño fue en bloques al azar con nueve repeticiones. El consumo de materia seca respondió de modo cuadrático, perjudicando el peso final, que disminuyó, con el aumento del nopal en la dieta. Aumentó el consumo de materia natural disminuyendo el de agua, el consumo total de agua aumentó en el tratamiento con mayor cantidad de nopal. La relación de consumos: agua voluntaria/ materia seca, disminuyó al aumentar el nopal. El nopal constituye una reserva de agua para ovinos Santa Inés en condiciones semiáridas de Brasil.

\section{SUMMARY}

The water intake in 45 male $(27.50 \pm 0.48 \mathrm{~kg}$ of mena body weight) Santa Ines sheep fed with increasing levels $(0,25,50,75,100 \%$ DM basis) of prickly pear (Opuntia ficus indica Mill) to replace corn was studied. A completely randomized block design with nine replications per treatment was used. Quadratic behavior was observed for the intake of dry matter, hitting the final weight, which decreased with increasing the cactus in the diet. There was an increase in the intake of natural material and a decrease in voluntary water intake;

Recibido: 17-8-10. Aceptado: 20-12-10.

\section{AdDitiOnAL KEYWORDS}

Cactus. Lambs. Production. Water deficit.

total water intake increased, and the ratio of intakes: water/dry matter, decreased in the treatment with larger cactus proportion. The pryckly pear constitues a water reservoir for Santa Inês sheep in Brazilian semiarid conditions.

\section{INTRODUCCIÓN}

El agua, frecuentemente es un factor limitante en la producción ganadera en áreas áridas y semiáridas, en donde los rebaños tienden a estar bajo condiciones de estrés debido a las altas temperaturas, alimentación de mala calidad y baja disponibilidad hídrica (Casamassima et al., 2008). Por ello, son necesarios recursos alternativos para sobrevivir a períodos críticos de escasez de alimento y agua (Véras et al., 2002). Tegegne et al. (2007) sugieren la utilización del nopal (Opuntia ficus indica Mill), por presentar características importantes de resistencia a la falta de agua, temperaturas elevadas, suelos pobres en nutrientes, pocos insumos energéticos y además de ser una planta de fácil manejo sus características nutritivas son favorables (Teixeira et al., 1999).

Además los ovinos alimentados con nopal fresco en condiciones de sequía pueden 
disminuir considerablemente el consumo de agua. El objetivo de este trabajo es determinar el consumo de agua en ovinos de la raza Santa Inés, en confinamiento, alimentados con diferentes niveles de nopal.

\section{MATERIAL E MÉTODOS}

El experimento fue realizado en la microrregión del Cariri Oriental Paraibano ( $7^{\circ} 29^{\prime} 34^{\prime \prime}$ de latitud sur y $36^{\circ} 41^{\prime} 53^{\prime \prime}$ de longitud oeste). El clima se clasifica de tipo Bsh'W (semiárido caliente) presentando temperaturas medias anuales de $26^{\circ} \mathrm{C}$ con pluviometría media anual de $395 \mathrm{~mm}$, con distribución irregular y estación seca de más de ocho meses.

Fueron utilizados 45 ovinos machos Santa Inés (peso de 27,35 $\pm 0,48 \mathrm{~kg}$ ), en corrales individuales con piso de tierra y comederos y bebederos individuales. El nopal fue picado en pedazos de aproximadamente cinco centímetros para facilitar la deglución. El nopal sustituyó al maíz en 0, $25,50,75$ y $100 \%$ de la materia seca, utilizándo como fuente de fibra Tifton-85 (Cynodon dactylon) triturado y mezclado con los ingredientes de las dietas (salvado de trigo, pasta de soya, maíz molido y sales minerales). Las dietas fueron formuladas (NRC, 1985) para ovinos de 25 kilogramos con ganancia diaria de peso de $250 \mathrm{~g}$.

Durante 45 días (10 días para adaptación), los alimentos ofertados y las sobras fueron pesados diariamente, para cálculo del consumo voluntario, estableciéndose $10 \%$ de sobras. El agua fue ofrecida ad libitum, pesando, tanto la oferta como las sobras de mañana y tarde.

Se empleó un diseño de bloques al azar formados por los animales según el peso inicial, con 5 tratamientos y 9 repeticiones. Se realizó el análisis de varianza y de regresión en función a los niveles de nopal en la dieta. Los análisis estadísticos fueron realizados a través de los procedimientos PROC GLM y PROC REG del SAS (1999).

\section{RESULTADOSYDISCUSIÓN}

La alimentación de ovinos de la raza Santa Inés con raciones conteniendo diferentes porcentajes de inclusión de nopal no presentó diferencia significativa entre el peso final de los animales. Para la ganancia de peso diario se observó una ligera disminución lineal $(\mathrm{p}<0,05)$ del tratamiento con $0 \%$ de nopal, para el tratamiento con $100 \%$ de inclusión (tabla I).

Para el consumo de materia natural se observó un aumento lineal $(\mathrm{p}<0,01)$, esto ocurrió debido a la alta palatabilidad que presenta el nopal, siendo éste preferido sobre los demás ingredientes (tabla II). El consumo de materia seca presentó un comportamiento cuadrático $(\mathrm{p}<0,05)$, teniendo un punto de máxima utilización de 54,18\% antes de perjudicar el consumo, con lo cual el consumo de la MS alcanza 1,49 kg/día.

El consumo de agua, a través del nopal aumentó linealmente $(p<0,01)$ con el aumento del nopal en la dieta. Este comportamiento fue inversamente proporcional al consumo de agua voluntario que disminuyó linealmente $(p<0,01)$, en torno de 25,6 gramos de agua/día por cada unidad porcentual de nopal en la dieta. Esta ingestión voluntaria redujo de 4,9 para $2,31 \mathrm{~kg}$ de agua/día, entre los tratamientos con 0 y $100 \%$ de inclusión del nopal, respectivamente. Esto se debió a la gran cantidad de agua que contiene el nopal, originando la disminución de ingestión de agua directa de los bebederos (Cavalcanti et al., 2008). Estos resultados corroboran los encontrados por Salem et al. (2004) y Costa et al. (2009), cuando estudiaron la inclusión del nopal en la dieta de ovinos y caprinos, reforzando la importancia de la utilización del nopal en regiones áridas y semiáridas, debido a que éste puede proporcionar economía de agua para las regiones donde el agua es escasa, afectando la producción animal (Silanikove, 2000).

Para el consumo total de agua aumentó linealmente $(p<0,01)$ con el aumento del no- 
CONSUMO DE AGUA DE OVINOS ALIMENTADOS CON NOPAL

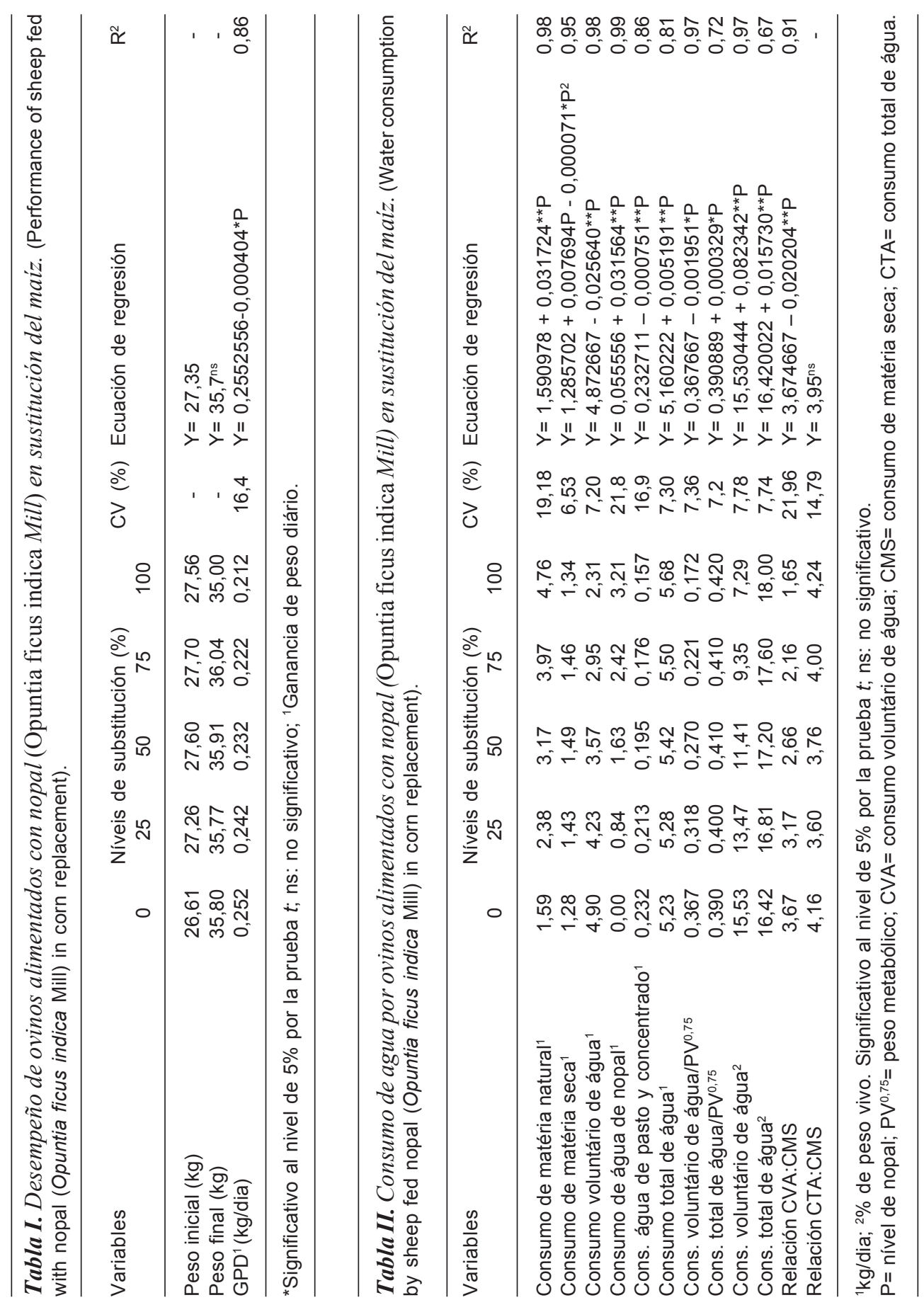

Archivos de zootecnia vol. 61, núm. 234, p. 303. 
pal en la dieta. Este aumento en la ingestión total de agua se debe al incremento de la humedad en la dieta, pues a cada unidad porcentual de la inclusión de nopal corresponde un incremento de aproximadamente 31,5 gramos de agua, resultando en $3,21 \mathrm{~kg}$ de agua para el nivel de $100 \%$ en los tratamientos en sustitución al maíz por el nopal, así como el atendimiento de la exigencias de agua por los animales, pues todos consumieron por encima de los $5 \mathrm{~kg}$ de agua por día. El comportamiento es muy similar al preconizado por el NRC (1985) que mediante una ecuación estima que por cada kilogramo de MS hay un consumo de 3,86 kg de agua/ día. El consumo de agua voluntaria por peso vivo metabólico, presentó un comportamien-

\section{BIBLIOGRAFÍA}

Casamassima, D., Pizzo, R., Palazzo, M., Alessandro, A.G. D' and Martemucci, G. 2008. Effect of water restriction on productive performance and blood parameters in comisana sheep reared under intensive condition. Small Ruminant Res, 78: 169-175.

Cavalcanti, C.V.A., Ferreira, M.A., Carvalho, M.C., Véras, A.S.C., Silva, F.M. e Lima, L.E. 2008. Palma forrageira enriquecida com uréia em substituição ao feno de capim tifton 85 em rações para vacas da raça Holandesa em lactação. Rev Bras Zootecn, 37: 689-693.

Costa, R.G., Beltrao Filho, E.M., Medeiros, A.N., Givisiez, P.E.N., Queiroga, R.C.R.E. and Melo, A.A.S. 2009. Effects of increasing levels of cactus pear (Opuntia ficus-indica L. Miller) in the diet of dairy goats and its contribution as a source of water. Small Ruminant Res, 82: 6265.

NRC. 1985. National Research Council. Nutrients requirements of sheep. National Academy Press. Washington, DC. 99 pp.

Salem, H.B., Nefzaoui, A. and Salem, L.B. 2004 Spineless cactus (Opuntia ficus indica $\mathrm{f}$. to lineal descendente y el consumo de agua total por peso vivo metabólico presentó un comportamiento lineal ascendente $(\mathrm{p}<0,05)$. El consumo de agua voluntaria y total de agua por el porcentaje de peso vivo presentaron un comportamiento lineal $(p<0,01)$. $\mathrm{La}$ relación de consumo de agua voluntaria por MS disminuyó linealmente $(\mathrm{p}<0,01)$.

\section{CONCLUSIÓN}

El nopal es una alternativa que reduce considerablemente el consumo de agua voluntaria en ovinos en confinamiento, siendo el nopal recomendado en la alimentación de ovinos en zonas áridas y semiáridas, donde el agua es escasa.

inermis) and oldmansaltbush (Atriplex nummularia L.) as alternative supplements for growing Barbarine lambs given straw-based diets. Small Ruminant Res, 51: 65-73.

SAS Institute. 1999. User's guide: Statistics, SAS. Cary, NJ.

Silanikove, N. 2000. The physiological basis of adaptation in goats to harsh environments. Small Ruminant Res, 35: 181-193.

Tegegne, F., Kijora, C. and Peters, K.J. 2007. Study on the optimal level of cactus pear (Opuntia ficus-indica) supplementation to sheep and its contribution as source of water. Small Ruminant Res, 72: 157-164.

Teixeira, J.C., Evangelista, A.R., Perez, J.R.O., Trindade, I.A.C. e De Moron, I.R. 1999. Cinética da digestão ruminal da palma forrageira (nopalea cochenillifera (I.) lyons-cactaceae) em bovinos e caprinos. Ciênc Agrotec, 23: 179-186.

Veras, R.M.L., Ferreira, M.A., Carvalho, F.F.R. e Véras, A.S.C. 2002. Farelo de Palma Forrageira (Opuntia ficus-indica Mill) em substituição ao milho, digestibilidade aparente de nutrientes. Rev Bras Zootecn, 31: 1302-1306. 